\title{
Assessing Medication Default, Alcohol Intake and Smoking Habits among Hypertensive Patients in a Rural Village of Limpopo Province, South Africa
}

Tugli A.K.

Department of Public Health, University of Venda, Thohoyandou, South Africa

Ramaano N.S.

Department of Correctional Services, Louis Trichardt, South Africa

Klu E.K.

Department of English, University of Venda, Thohoyandou, South Africa

Mokonoto M.

Gauteng Provincial Legislature, South Africa

Korkpoe C.H.

Department of Statistics, University of Venda, Thohoyandou, South Africa

Morwe K.

Institute for Gender \& Youth Studies, University of Venda, Thohoyandou, South Africa

\section{Doi:10.5901/mjss.2014.v5n23p1614}

Abstract

Hypertension (high blood pressure), though treatable and manageable, is a major risk factor for cardiovascular and renal diseases. The study assessed medication compliance, alcohol intake and smoking habits among hypertensive patients in a rural village of Limpopo province. A descriptive cross-sectional design was employed where self-reported questionnaires were used to collect data. A total of 89 hypertensive patients participated in the study giving a $97.8 \%$ response rate. Most $(n=75$; 84.3\%) participants were females and the average ages for males and females were $44.14 \pm 9.08$ years and $46.49 \pm 8.05$ years respectively. Most participants ( $n=70 ; 78.7 \%$ ) were divorced; about a quarter ( $n=23 ; 25.8 \%$ ) had no formal schooling and 40(44.9\%) were unemployed. Furthermore, this study found that $13.5 \%$ were smokers, $20.2 \%$ consumed alcohol and $20 \%$ reported not to be complying with their antihypertensive medication regimen. In addition, the study found statistically significant differences ( $p=0.000$ and $p=0.025$ ) between male and female smokers and the mean number of bottles of alcohol consumption respectively. There is a need to heighten hypertension awareness and management through health education by targeting low socio-economic communities.

Keywords: hypertensive patients, medication default, alcohol intake, smoking habits, rural village.

\section{Introduction and Background of the Study}

Hypertension or high blood pressure measured in millimeters of mercury $(\mathrm{mm} \mathrm{Hg})$, is generally considered as blood pressure reading $\geq 140 \mathrm{~mm} \mathrm{Hg}$ (systolic) or $\geq 90 \mathrm{~mm} \mathrm{Hg}$ (diastolic)(University of Maryland Medical Center(UMMC), 2013). Hypertension puts a lot of strain on organs such as the hearts and kidneys, and contributes to $75 \%$ of all strokes and heart attacks. Besides the morbidity and mortality aspects, it has public health and other socio-economic implications. It is projected that the number of deaths to be attributed to hypertension over the next 20 years may well exceed substantially the number resulting from HIVIAIDS (Lloyd-Sherlock et al., 2014).

Despite the fact that there are a number of risk factors associated with hypertension, Weber (2014), Appel (2003) and Gascón, et al. (2004) posit that, it can be managed through pharmacologic and non-pharmacologic or lifestyle 
modifications. According to Tomson and Lip (2005), the relationship between alcohol and cardiovascular disease, especially hypertension and coronary heart disease, is not as clear-cut; however, for most diseases related to alcohol consumption, a dose-response effect exists with risk of the disease increasing with greater amounts of alcohol intake. That notwithstanding, Klatsky (1995) and Marmot et al. (1994) in Tomson and Lip (2005) allude that both cross-sectional and prospective epidemiological studies have established a significant relationship between hypertension and alcohol consumption.

In their study to test the hypothesis that the action of alcohol on blood pressure is reversible and that its effect is mainly due to very recent alcohol consumption, Maheswaran et al. (1991) established that the effect of alcohol on blood pressure appears to be predominantly due to alcohol consumed in the few days immediately preceding blood pressure measurement. In line with this assertion, Kenigsberg (2011) argues that alcohol induced hypertension may be the most prevalent form of secondary hypertension. This source cited Nakamura et al. (2007) as stating that a recent study attributed alcohol as the cause of hypertension in $34.4 \%$ men and $2.6 \%$ women in a Japanese population.

According to Sheps (2012), too much alcohol intake does not only raise blood pressure to unhealthy levels but can also interfere with the effectiveness and increase the side effects of some blood pressure medications. He maintains that an alcoholic drink which is 12 ounces(355milliliters) of beer, 5 ounces(148 milliliters) of wine or 1.5 ounces (44 milliliters) of 89-proof distilled spirits contains calories that may contribute to unwanted weight gain - a risk factor for high blood pressure. Moderate drinking is generally two drinks a day for men younger than 65 years, one drink a day for men 65 years and older or one drink a day for women of any age.

The effect of cigarette smoking on individual's health has become a public health issue. To this end, Weber (2014) emphasizes that smoking is regarded as a major risk factor for high blood pressure and overall cardiovascular diseases; and, in addition, it remains a significant risk for respiratory diseases and cardiovascular mortality. Not only does smoking or chewing tobacco immediately raise blood pressure temporarily, but the chemicals in tobacco can damage the lining of the artery walls. This can cause arteries to narrow, thus increasing blood pressure (Coetzee, 2010; UMMC, 2013). In a nationally representative sample of adults aged 16 years and older in a health survey for England, Primatesta et al. (2000) found that older male smokers had higher systolic blood pressure than did nonsmoking men; and among women, light smokers (1-9cigarettes per day) tended to have lower BPs than heavier smokers and never smokers.

Despite the availability of effective therapy, hypertension remains poorly controlled due to non-compliance (Appel, 2003; Osamor \& Owumi, 2011). Compliance involves the degree to which patients conform to medical advice, comply with lifestyle and dietary changes and keeping medical appointment and follow-ups and taking and sticking to treatment prescription and regimen. Non compliance to blood pressure-lowering treatment is a major reason for poor control of high blood pressure worldwide. Although safe and effective drugs are available, the management of high blood pressure is still far from optimal especially in developing countries (Osamor \& Owumi, 2011).

In the Third National Health and Nutrition Examination Survey in the US, Burt et al.(1995) in Wang et al.(2002), reported that nearly half of hypertensive patients in their community-based sample were found to be taking no prescription medication, and only a quarter of those who were being treated had their blood pressure adequately controlled. In another community-based cross-sectional study involving 440 hypertensive patients, Osamor and Owumi (2011) found that though $77.5 \%$ of the participants complied with keeping their follow-up appointments every time, $41.5 \%$ had poor self-reported compliance at different levels ranging from regularly missing to take their medication to rarely taking medication. For factors affecting medication compliance, Mahmoud (2012) identified sex, educational level, work status, smoking habits etc. Other associated factors with inadequate treatment of hypertension include reservations and beliefs about efficacy of drugs, lack of necessary knowledge of the condition and its treatment and side effects (Primatesta et al. 2000; Gascón, et al., 2004; Kabir et al., 2004).

Addressing the issue of hypertension requires both pharmacologic and non-pharmacologic approaches on the part of the patient. This must translate into pro-active living, lifestyle modification and medication compliance. In the light of this, the study assessed medication default, alcohol intake and smoking habits among hypertensive patients in a rural setting.

\section{Methodology}

\subsection{Study design}

This was a descriptive study using a cross-sectional quantitative design. The design was best suited because it answered the research questions under investigation. 


\subsection{Study population}

The study was conducted in the Folovhodwe village. The target population included 91adult patients who were diagnosed with high blood pressure and were taking anti-hypertensive treatment at Folovhodwe clinic in the Mutale municipality of the Limpopo province.

\subsection{Instrument and data collection}

Self-reported questionnaire, in both English and the local Vha-Venda languages, was used to collect data. It was designed to solicit information pertaining to socio-demographic characteristics (age, gender, marital status, employment, educational level etc), patients' adherence to medication, alcohol intake and smoking habits. Variables such as medication default, alcohol intake and smoking status were assessed using "yes" or " no" binary option questions. The instrument was pre-tested through a random selection of five participants from a similar setting using. The study was conducted over four weeks between June and July 2012 after identifying the subjects with high blood pressure from the clinic register. Dates and choice of venue for the administration of the questionnaires were negotiated with the participants.

\subsection{Ethical consideration}

Data collection commenced after the approval certificate (SHS/11/PH/06/0811) issued by the Research and Innovation Directorate of the University of Venda. Permissions to conduct the research and to gain access to the clinic were also obtained from the Department of Health (Reference 4/2/2 in May 2012) - Limpopo province and the official in charge of the selected clinic. Finally, access to the study site was negotiated with the headman of the community. Participation in this study was voluntary and all participants were requested to give informed consent before the study was conducted. Finally, all participants were assured of confidentiality of their responses and anonymity of their identities.

\subsection{Data analysis}

Data were analysed using the Statistical Package for Social Sciences (SPSS) version 17. The analysis involved descriptive statistics where percentages, frequency tables, etc were used. Whilst the two sample t-test was used to compare the means of alcohol consumption per day with respect to male and female participants, the Pearson $\mathbf{X}^{2}$ (Chisquare) test was used to establish if any association exists between male and female with respect to smoking. P-value $\leq$ 0.05 was used as the cut-off point for establishing statistical significance.

\subsection{Results}

\subsubsection{Socio-demographic characteristics}

A total of 89 hypertensive patients participated in the study giving a response rate of $97.8 \%$. Of this figure, an overwhelming proportion $(n=75 ; 84.3 \%)$ of them were females. Almost half $(48.3 \%)$ of the participants were between 41 50 years. The average ages for males and females were $44.14 \pm 9.08$ years and $46.49 \pm 8.05$ years respectively. Most participants ( $n=70 ; 78.7 \%)$ were divorced and about 1 in $5(20.2)$ was married. Whist about a quarter $(n=23 ; 25.8 \%)$ of the participants had no formal schooling, 18 (20.2\%) attended primary school, 28 (31.5\%) attended secondary school and 20 (22.5\%) attended tertiary institutions. As regards their employment status, 40(44.9\%) were unemployed, 28 (31.5\%) were employed, 15 (16.9\%) were self employed and 6 (6.7\%) were pensioners.

\subsubsection{Smoking among participants}

As shown in Table 1, 12(13.5\%) of the participants were smokers. Whilst 1 in 2 males smokes, an overwhelming majority of the females ( $n=70 ; 86.5 \%)$ were non-smokers. Among the participants 1 in 2 male participants and only $4(5.5 \%)$ of female participants reported to be smoking. Furthermore, the Pearson's Chi-square test was used to establish if there is any relationship between the two categorical variables (gender and smoking status). The $p$-value of $0.000<0.05$ signifies that there was statistically significant difference between male and female smokers at the $5 \%$ level. 
Table 1: Distribution of smoking status across gender

\begin{tabular}{|c|c|c|c|c|}
\hline \multirow[b]{2}{*}{ Smoking status } & \multicolumn{2}{|c|}{ Gender } & \multirow{2}{*}{ Total } & \multirow{2}{*}{ Test statistics } \\
\hline & Male n(\%) & Female $\mathrm{n}(\%)$ & & \\
\hline Yes & $7(50 \%)$ & $5(6.7 \%)$ & $12(13.5 \%)$ & \multirow{3}{*}{$\begin{array}{c}x^{2} \text { (Chi-sq) }=18.991 \\
d f=1 \\
p=0.000<0.05\end{array}$} \\
\hline No & $7(50 \%)$ & $70(93.3 \%)$ & $77(86.5 \%)$ & \\
\hline Total & $14(100 \%)$ & $75(100 \%)$ & $89(100 \%)$ & \\
\hline
\end{tabular}

\subsubsection{Alcohol consumption among participants}

Among the participants a total of $18(20.2 \%)$ reported drinking alcohol. Whilst a greater proportion of males (57.1\%) than females (13.3\%) indicated that they drink alcohol, males and their female counterparts on the average consume 7.63 and 4.50 bottles per day respectively (Table 2). The independent t-test was used to establish whether there was a significant difference between the mean alcohol consumption (number of bottles consumed per day) and gender. At the $5 \%$ level of significance, there was a statistically significant difference between alcohol consumption with males tending to consume more than females $(p=0.025)$.

Table 2: Distribution of alcohol intake across gender

\begin{tabular}{|l|c|c|l|}
\hline \multirow{2}{*}{ Alcohol intake } & \multicolumn{2}{|c|}{ Gender } & Test statistics (independent two \\
\cline { 2 - 3 } & Male(N=14) & Female(N=75) & sample t-test) \\
\hline $\mathrm{n}(\%)$ & $8(57.1 \%)$ & $10(13.3 \%)$ & T-statistic $=-2.349$ \\
$\begin{array}{l}\text { Average number of bottles consumed on daily basis(mean } \pm \\
\text { standard deviation) }\end{array}$ & $7.63 \pm 3.249$ & $4.50 \pm 2.121$ & $\begin{array}{l}\mathrm{df}=11.545 \\
\mathrm{p}(\text { sig. 2-tailed) }=0.025\end{array}$ \\
\hline
\end{tabular}

\subsubsection{Medication default among participants}

Figure 1 depicts the level of compliance to anti-hypertensive treatments. Whilst $71(80 \%)$ of the participants reported that they comply with their medication regimen, $18(20 \%)$ were not.

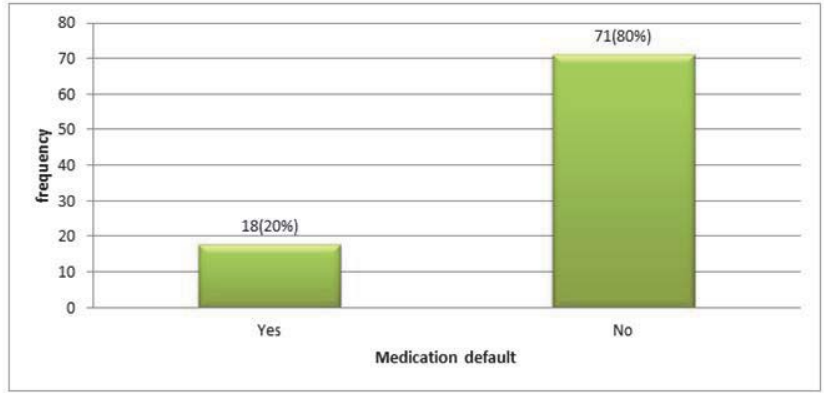

Figure 1: Level of medication default among the study participants

Among the reasons adduced by the non-compliant patients were:

- Forgetfulness

- No interest

- Fear of side effects

- Lack of knowledge about hypertension

- Use of alternative traditional medication

- Lack of means to the local clinic

\section{Limitation of the Study}

One of the limitations of the study is that the variables of interest were sensitive and measured using self-report 
instrument. This could influence the participants' responses. Secondly, the study focused on patients from only one rural health facility with very few hypertensive patients and as such broad generalizations can not be supported.

\section{Discussion}

The study assessed medication default, alcohol intake and smoking habits among hypertensive patients at Folovhodwe village in the Limpopo Province. The participants' socio-demographic and economic contexts reveal that the average ages for males and females were $44.14 \pm 9.08$ years and $46.49 \pm 8.05$ years respectively; $84.3 \%$ were females, $78.7 \%$ were divorced, $44.9 \%$ were unemployed whilst about 1 in 4 participants (25.8\%) had no formal schooling. The socioeconomic background of the participants, indeed, depicts a pathetic outlook as low level of education and employment can influence knowledge, beliefs and attitudes regarding positive health outcomes decisions (Mahmoud, 2012; Osamor and Owumi, 2011). According to Morenoff et al. (2007), Dirksen et al.(2007) and Zungu et al.(2013), social, economic and environmental contexts in which people have a great potential of influencing individuals' knowledge, attitude, practices and lifestyle/habits. Undoubtedly, such a high divorce rate of $78.7 \%$ and $44.9 \%$ unemployment rate among the participants in this study can trigger unhealthy social habits like smoking, drinking and drug abuse. Subscribing to this notion, Pharr et al. (2012) posit that unemployment is associated with unhealthy behaviours; and study conducted by Atallah et al. (2007) in the Caribbean, indicate that the high prevalence and poor control of hypertension have been observed in populations with low-socioeconomic status including poor therapeutic control in lower social classes.

In the present study, whilst $13.5 \%$ of the participants were smokers, there is a significant relationship $(\mathrm{p}=0.000)$ between one' gender and smoking (Table 1). This smoking prevalence rate is more than three times found among hypertensive patients attending a primary health care clinic in Botswana by Zungu et al.(2013). This source further states that among the smokers, 29.4\% smoked between 6 to 10 cigarettes per day whilst 5.8\% smoked more than 10 per day. The link between smoking and hypertension is no longer in question. Cigarette smoking is regarded as a major risk factor for high blood pressure and overall cardiovascular diseases, and it remains a significant risk for respiratory diseases and cardiovascular mortality (Dochi et al., 2009; Weber, 2014). Seemingly in the current study, some hypertensive patients tend to be oblivious of the aggravating effect of heavy smoking on their current condition.

The study also found that about 1 in 5 participants (20.2\%) consumed alcohol. Whilst males consumed 7.63 per day on the average, their female counterparts consumed 4.50 bottles which showed a significant difference ( $t=-2.349$; $\mathrm{p}=0.025$ ) between male and female average consumption per day (Table 2). As far as alcohol is concerned, Sheps (2012) points out that it contains calories that may contribute to unwanted weight gain which is an added risk factor for high blood pressure. Furthermore in this study, among those who consume alcohol, their consumption level is far higher (average 4.5 females and 7.63 for males) than the normal average of three drinks or less (Sheps, 2012). This scenario especially among hypertensive patients can be a recipe for disaster because alcohol can interfere with the efficacy of some blood pressure medications and can also contribute to medication default. Since it is established that cessation of heavy alcohol consumption leads to a reduction in 24 hour ambulatory systolic blood pressure and diastolic blood pressure of 7.2 and $6.6 \mathrm{mmhg}$, respectively (Pisoni et al., 2009), it will be medically prudent for hypertensive patients to resort to moderate drinking or quit alcohol intake completely.

One in five $(20 \%)$ participants in this study was found to be medically non-compliant with their hypertensive medications. Compliance involves the degree to which patients conform to medical advice, comply with lifestyle and dietary changes and keeping medical appointment and follow-ups and taking and sticking to treatment prescription and regimen. According to Osamor and Owumi (2011), safe and effective drugs are available for effective control of hypertension but, the management of high blood pressure is still far from optimal which the finding of this study has shown. Though in their studies, Burt et al.(1995) in Wang et al.(2002) and Osamor and Owumi (2011) less than the close to $50 \%$ and $41.5 \%$ non-compliance among their participants respectively, pharmacological default in any way can be costly and deadly with serious public health implications. Some of the reasons adduced by participants in this study for non-compliance included forgetfulness, fear of side effects, lack of knowledge about hypertension, use of alternative traditional medication etc. Similar findings were reported in studies by Primatesta et al. (2000), Gascón, et al. (2004) and Kabir et al. (2004). To this end, Thrall et al. (2004) acknowledges that social support from the families and health professionals can enhance medication compliance.

Lack of knowledge about hypertension, being cited as one of reasons for medication default, can be a critical drawback in terms of understanding the condition, its signs and symptons including proactive behavior and lifestyle changes. According to Zungu et al. (2013), knowledge is a crucial determinant of behavior change and lifestyle practices. Appel (2003) argues that lifestyle modification or non-pharmacologic therapy has important roles in hypertensive as well as non hypertensive individuals. Smoking, alcohol and medication default, as found in this study, can be a dangerous risk 
elevation factor for hypertension. This situation thus warrants urgent lifestyle modification which according to Appel(2003) can serve as initial treatment before the start of drug therapy and as an adjunct to medication in persons already on drug therapy.

\section{Conclusion}

Hypertension, on one hand, is preventable whilst at the same time a manageable condition. Among the hypertension patients in this study, $13.5 \%$ were found to be smokers, $20.2 \%$ consumed alcohol and $20 \%$ reported not complying with their antihypertensive medication regimen. Findings such as these point to some underlying socio-economic and environmental factors requiring an in-depth qualitative investigation in order to bring to the fore the patients' point of view, needs and priorities pertaining to the reality of living with hypertension. It further dictates that management of high blood pressure is not only about pharmacologic therapy but also about lifestyle modification, sticking to rigid dietary regimen coupled with strong will power. This study, therefore, recognizes the need for action because the consequences of hypertension can be devastating with far reaching consequences in terms cost, morbidity, mortality as well as social burden that can be borne by families and communities.

\section{Recommendation}

Recognizing the fact that the consequences of hypertension can be devastating and far reaching in terms cost, morbidity, mortality as well as the social burden that it places on families and communities, this study, therefore, recommends the following:

- The need to heighten to hypertension awareness through health education targeting low socio economic communities in areas such as knowledge and management of hypertension

- Communities and families must be capacitated to provide social network support system for hypertensive patients

- Good communication between health service providers and hypertensive patients should be promoted.

\section{References}

Appel,L.J. (2003). Lifestyle modification as a means to prevent and treat high blood pressure. Journal of American Society of Nephrology, Vol.14 no. supplementary 2, S99-S102.

Atallah, A., Inamo, J., Larabi, L., Chatellier, G., Rozet, JE., Machuron, C., De Gaudemaris, R.\& Lang, T. (2007). Reducing the burden of arterial hypertension: what can be expected from an improved access to health care? Journal of Human Hypertension, 21,316322.

Burt, V., Whelton, P., Roccells, E., et al. (1995). Prevalence of hypertension in the US adult population: results from the Third National Health and Nutrition Examination Survey. In: Wang, P.S., Bohn, RL., Knight, E., Glynn, R.J., Mogun, H. \& Avorn, J. (2002). Noncompliance with Anthypertensive medications: the impact of depressive symptoms and psychological factors. Journal of General Internal Medicine, 17(7), 504-511.

Coetzee, K. (2010). Types of hypertension. Available at: http://www.health24.com/Medical/Hypertension/Types-of-blood-pressure/Typesof-hypertension-20120721. (Accessed 8 May 2011).

Dirksen, S.R., O'Brian, P.G., Lewis, S.L., Heitkemper, M.M \& Bucher, L. (2007). Clinical companion medical-surgical nursing. (7th Ed). St. Louis, Missouri: Elsevier.

Dochi, M., Sakata, K., Oishi, M., Tanaka, K., Kobayashi, E. \& Suwazono, Y. (2009). Smoking as an independent risk factor for hypertension: a 14-year longitudinal study in male Japanese workers. Tohoku Journal of Experimental Medicine, 217(1), 37-43.

Gascón,J.J., Sánchez-Ortuño, M., Llor, B., Skidmore, D. \& Saturno, P.J. (2004). Why hypertensive patients do not comply with the treatment: Results from a qualitative study. Family Practice, 21, 125-130.

Kabir, M., Illiyasu, Z., Abubakar, I.S. \& Jibril, M. (2004). Compliance to medication among hypertension patients in Murtala Mohammed specialist hospital, Kano, Nigeria: Journal of Community Medicine and Primary Health Care, 16(1), 16-20.

Kenigsberg, B. (2011). How does alcohol affect hypertension? New York: University of New York.

Klatsky, A.I. (1995). Blood pressure and alcohol intake. In: Tomson, J. and Lip, G.Y.H. (2005). Alcohol and hypertension: an old relationship revisited. Editorial comment. Birmingham: Oxford University Press.

Lloyd-Sherlock, P., Ebrahim, S. \& Grosskurth. (2014). Is hypertension the new HIV epidemic? International Journal of Epidemiology,doi: 10.1093/ije/dyu019.

Maheswaran, R., Gill, J.S., Davies, P. \& Beevers, D.G. (1991). High blood pressure due to alcohol: a rapidly reversible effect. Hypertension, 17(6), 787-792.

Mahmoud, M.I.H. (2012). Compliance with treatment of patients with hypertension in Almadinah Almunawwarah: A community-based study. Journal of Taibah University Medical Sciences, 7(2), 92-98. 
Marmot, M.G., Elliott, P., Shipley, M.J. et al. (1994). Alcohol and blood pressure.In: Tomson, J. and Lip, G.Y.H. (2005). Alcohol and hypertension: an old relationship revisited. Editorial comment. Birmingham: Oxford University Press.

Morenoff, J.D., Hansen, B.B., House, J.S., Williams, D.R., Kaplan, G.A. \& Hunter, H.E.(2007). Understanding social disparities in hypertension prevalence, awareness, treatment and control: The role of neighbourhood context. Social Science Medicine, 65(9), 1853-1866.

Nakamura, K., Okamura, T., Hayakawa, T, et al. (2007). The proportion of individuals with alcohol-induced hypertension among total hypertensives in a general Japanese population: NIPPON DATA 90. In: Kenigsberg, B. (2011). How does alcohol affect hypertension? New York: University of New York.

Osamor, P.E. \& Owumi, B.E. (2011). Factors associated with treatment compliance in hypertension in South west Nigeria. Journal of Health, Population and Nutrition, 29(6), 619-628.

Pharr, J.R., Moonie, S. \& Bungum, T.J. (2012). The impact of unemployment on mental and physical, access to health care and health risk behavior. Public Health, 2012, 483432,7 pages, doi:10.5402/2012/483432.

Pisoni, R., Mustafa, I.A. \& Calhoun, D.A. (2009). Characterization and Treatment of Resistant Hypertension: National Institutes of Health, 11(6), 407-413.

Primatesta, P., Falaschetti, E., Gupta, S., Marmot, M.G. \& Poulter, N.R. (2000). Association between smoking and blood pressure. Evidence from the Health survey for England. London: Department of Health (England).

Sheps, S.G. (2012). High blood pressure (hypertension). Minnesota: Mayo Foundation for Medical Education and Research.

Soardi, G., Donnini, D., Moretti, M., Milocco, C., Catena, C. \& Sechi, L.A. (2008). Effects of antihypertensive drugs on alcohol-induced functional responses of cultured human endothelial cells. Hypertension Research, 31, 345-351.

Tomson, J. \& Lip, G.Y.H. (2005). Alcohol and hypertension: an old relationship revisited. Editorial comment. Birmingham: Oxford University Press.

University of Maryland Medical Center (UMMC) (2013). High blood pressure. Available at: http://umm.edu/health/medical/reports/articles/ high-blood-pressure\#ixzz335WOb2uo. (Accessed 15 May 2014).

Wang, P.S., Bohn, R.L., Knight, E., Glynn, R.J., Mogun, H. \& Avorn, J. (2002). Noncompliance with Antihypertensive medications: the impact of depressive symptoms and psychological factors. Journal of General Internal Medicine, 17(7), 504-511.

Weber, C. (2014). Top 10 high blood pressure risk factors. Available at: http:/highbloodpressure.about.com/od/understandingyourrisk/ tp/risk_tp.htm (Accessed 25 April 2014).

Zungu, L.I., Djumbe, F.R. \& Setswe, K.G. (2013). Knowledge and lifestyle practices of hypertensive patients attending a primary health care clinic in Botswana. African Journal for Physical, Health Education, Recreation and Dance (Supplement 1), November, 123 138. 